\title{
Vortex dynamics in Permalloy disks with artificial defects: Suppression of the gyrotropic mode
}

\author{
K. Kuepper, ${ }^{\text {a) }}$ L. Bischoff, Ch. Akhmadaliev, and J. Fassbender \\ Forschungszentrum Dresden-Rossendorf, Institute of Ion Beam Physics and Materials Research, \\ P.O. Box 5101 19, D-01314 Dresden, Germany \\ H. Stoll, K. W. Chou, A. Puzic, K. Fauth, D. Dolgos, and G. Schütz \\ Max-Planck-Institut für Metallforschung, D-70569 Stuttgart, Germany \\ B. Van Waeyenberge \\ Department of Subatomic and Radiation Physics, Ghent University, 9000 Ghent, Belgium \\ T. Tyliszczak \\ Advanced Light Source, LBNL, Berkeley, California 94720 \\ I. Neudecker, G. Woltersdorf, and C. H. Back \\ Experimentelle Physik, Universität Regensburg, D-93053 Regensburg, Germany
}

(Received 29 September 2006; accepted 19 December 2006; published online 6 February 2007)

\begin{abstract}
The dynamics of magnetic vortices in thin Permalloy disks having artificial defects in the form of small holes at different locations within the disk has been investigated by means of frequency-domain spatially resolved ferromagnetic resonance. It is found that the vortex can be effectively captured by such a defect. Consequently the commonly observed gyrotropic vortex motion in an applied microwave field of $1 \mathrm{mT}$ is suppressed. However, if in addition a static magnetic field of at least $4.3 \mathrm{mT}$ is applied, the vortex core is nucleated from the artificial defect and a modified gyrotropic motion starts again. (C) 2007 American Institute of Physics.
\end{abstract}

[DOI: $10.1063 / 1.2437710]$

In the quest for magnetic data storage devices at the nanoscale, the study of the static and dynamic properties of thin magnetic films has attracted much attention in the last years. These structures usually tend to form planar magnetic domain configurations. One special magnetization configuration is the so-called vortex structure, i.e., the spin in the center of an in-plane oriented magnetization curl points perpendicular to the plane. Although theoretically predicted a long time ago, ${ }^{1,2}$ the vortex core was experimentally observed only recently by means of magnetic force microscopy ${ }^{3}$ and spin polarized tunneling microscopy. ${ }^{4}$ Since time-resolved measurement techniques with high lateral resolution became available, ${ }^{5-7}$ the magnetic excitations of such vortex structures were investigated in the time regime. Especially the so-called gyrotropic mode, a sub gigahertz characteristic excitation of the magnetic vortex core itself, is of enormous interest. ${ }^{8-14}$

The numerous efforts to study the gyrotropic mode in depth stem partly from the importance to understand the fundamental magnetic properties of these magnetic nanostructures, and partly from the technological challenge to achieve a controllable and reproducible switching behavior of the vortex core. Another possibility to influence the behavior of a vortex core is the insertion of artificial defects, e.g., small holes, into the magnetic structures. It has been predicted theoretically ${ }^{15}$ and also found experimentally ${ }^{16,17}$ that such small defects can attract and capture magnetic vortices, thus one may switch the vortex between such artificial defects. The shifting and nucleation of a vortex in an applied magnetic field has been demonstrated recently by means of Lorentz microscopy. ${ }^{18}$ However, up to now the magnetization

\footnotetext{
${ }^{a)}$ Electronic mail:k.kuepper@fzd.de
}

dynamics in the presence of small artificial defects and applied magnetic fields has not been addressed. This is the main goal of the present letter.

We apply spatially resolved ferromagnetic resonance (SR-FMR) in combination with scanning transmission x-ray microscopy (STXM). ${ }^{11}$ This technique offers unique capabilities to study and image the magnetization dynamics of the vortex gyrotropic motion. Moreover, the application of external static fields up to a few hundreds of oersteds is possible.

The samples investigated were $50 \mathrm{~nm}$ thick Permalloy disks of $1.5 \mu \mathrm{m}$ (1), $2 \mu \mathrm{m}$ (2), and $3 \mu \mathrm{m}$ (3) diameters, which have been prepared on top of a microwave compatible transmission line $(100 \mathrm{~nm} \mathrm{Cu}$ electrically isolated by a thin $\mathrm{Al}_{2} \mathrm{O}_{3}$ layer) in a multistep lithography and lift-off process. ${ }^{10}$ In order to obtain a reasonable transmission of the incident $\mathrm{x}$-rays in a thin $\mathrm{Si}_{3} \mathrm{~N}_{4}$ membrane was used as a substrate. Artificial small holes of about $300 \mathrm{~nm}$ diameter have been introduced in samples (2) and (3) by means of $\mathrm{Co}^{2+}$ focused ion beam erosion with ion fluences of about $10^{17}$ ions $/ \mathrm{cm}^{2}{ }^{2}{ }^{19}$ In disk (3) the hole was located in the center of the disk whereas in disk (2) an eccentric position was chosen. For comparison, disk (1) was not modified (Fig. 1).

The SR-FMR was performed using the STXM (Ref. 20) located at beam line 11.02 of the Advanced Light Source. Element specific x-ray magnetic circular dichroism was used as the contrast mechanism. ${ }^{21}$ The excitation energy of the incoming x-ray photons was set to the $\mathrm{Ni} L_{3}$ edge in order to maximize the magnetic contrast. An incidence angle of $30^{\circ}$ with respect to the sample normal was chosen to visualize the in-plane magnetization. The experimental setup used is identical to that described by Van Waeyenberge et al. ${ }^{14}$ Time resolution is achieved by using a high-frequency continuous wave generator which is synchronized with respect to the 


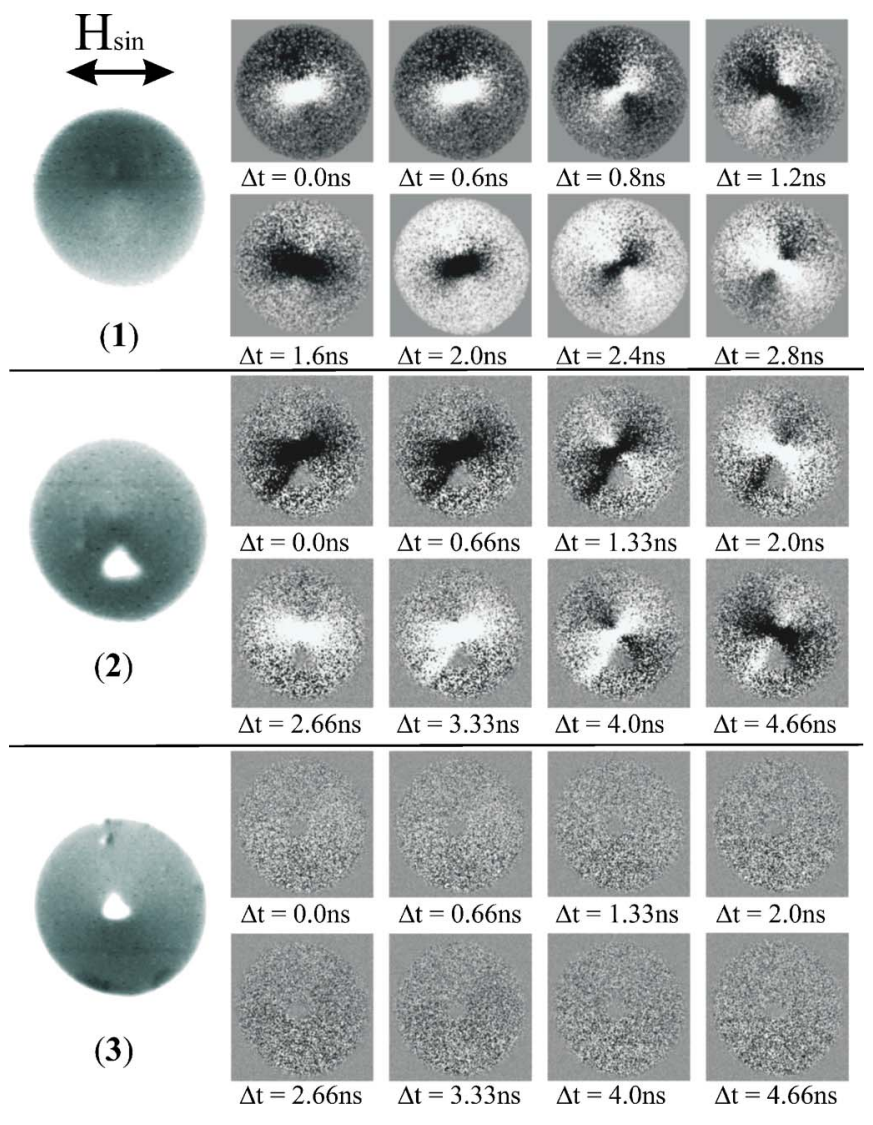

FIG. 1. (Color online) Left panel: Static images at the Ni $L_{3}$ absorption edge of the investigated Permalloy disks (1)-(3). Right panel: SR-FMR differential images taken at $45^{\circ}$ phase steps; the corresponding time delays are noted. FMR sequence recorded on disk (1) (top), disk (2) (center), and disk (3) (bottom) respectively (see text for more details). The samples are excited by a sinusoidal in-plane magnetic field $H_{\text {sin }}$.

$\mathrm{x}$-ray flashes from the synchrotron. By exciting the sample with such a continuous in-plane rf sine wave, one can observe the magnetic vortex movement at subgiahertz frequencies. By means of fast gating, eight channels are recorded within one microwave cycle allowing for a direct differential imaging in $45^{\circ}$ phase steps. For further details see Refs. 11 and 14. Dependent on the sample size (number of pixels) and the dwell time per pixel (between 10 and $40 \mathrm{~ms}$ ) we averaged over about $10^{10}$ bunches to acquire one image.

On the left hand side of Fig. 1 the magnetic images of the three Permalloy disks are shown. The magnetization directions in the top and bottom regions are aligned antiparallel to each other, which is reflected by the light and dark gray contrast arising from a magnetization component either parallel or antiparallel to the photon propagation direction. The left and right sides are imaged in an intermediate gray due to a vanishing magnetization component along the photon propagation direction. In disk (1) the direction of flux closure of the vortex structure is opposite to those in (2) and (3). The locations of the holes in disks (2) and (3) are clearly visible due to reduced absorption.

In the right panel of Fig. 1 differential image sequences are shown. For disks (2) and (3) an excitation frequency of 187.5 MHz with a field amplitude of approximately $1 \mathrm{mT}$ is used. Since the frequency of the gyrotropic motion is higher for smaller disks with the same film thickness, an excitation frequency of $312 \mathrm{MHz}$ is chosen for disk (1). According to the two-vortex side charges analytical model, which explicDownloaded 31 Mar 2007 to 132.199.211.1. Redistribution subjec

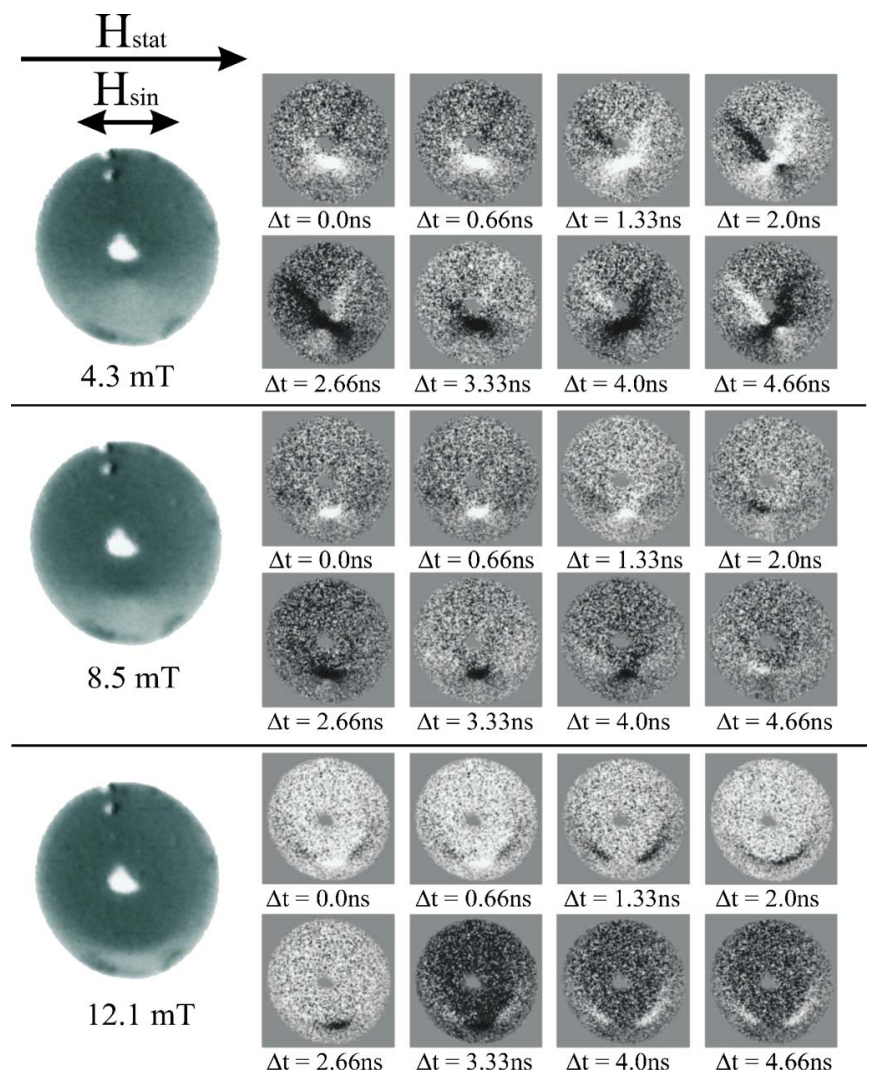

FIG. 2. (Color online) Disk (3): Nucleation of the vortex and gyrotropic vortex core motion at an excitation frequency of $187.5 \mathrm{MHz}$ in different applied static magnetic fields. Left panel: static STXM images; right panel: the gyrotropic vortex core motion in applied static fields of $4.3 \mathrm{mT}$ (top), $8.2 \mathrm{mT}$ (center), and $12.1 \mathrm{mT}$ (bottom). In addition to the sinusoidal field an in-plane external field $H_{\text {stat }}$ was applied in order to nucleate and displace the vortex.

itly accounts for the dot magnetostatic energy, ${ }^{22}$ the vortex gyrotropic modes are $333.5 \mathrm{MHz}$ [disk (1)], $249 \mathrm{MHz}$ [disk (2)], and $164.5 \mathrm{MHz}$ [disk (3)], assuming a saturation at magnetization $M_{S}=800 \mathrm{kA} / \mathrm{m}$. These frequencies have been found to be about $10 \%-15 \%$ higher than those obtained in corresponding experiments. ${ }^{23}$ Nevertheless, this model gives a good approximation of the vortex gyrotropic mode frequency in thin Permalloy disks. In the case of disks (1) and (2) the differential images comprise black and white patterns in the shape of dots and crosses which can be interpreted as a gyromagnetic motion of the magnetic vortex core. ${ }^{11}$ In the case of disk (2), where the artificial defect (hole) is located well below the sample center in the bottom domain, the vortex core motion is hardly affected by the presence of the defect. The distance between the vortex core and the defect is too large to account for a direct interaction between the defect and the vortex motion. The reversed contrast observed for sample (2) as compared to (1) is due to the opposite direction of flux closure of the vortex structure. In the case of disk (3) the hole is located in the center of the disk. Here the vortex is captured in the hole and the magnitude of the microwave field is too small to nucleate the vortex core. Thus the gyrotropic mode is completely suppressed.

Since the microwave field amplitude could not be increased sufficiently to nucleate a vortex core from the trap an additional static magnetic field is used (Fig. 2). By varying the applied field it is found that a static field of $4.3 \mathrm{mT}$ is necessary to nucleate a vortex, in good agreement with the
to AIP license or copyright, see http://apl.aip.org/apl/copyright.jsp 
results obtained by Uhlig et al. ${ }^{18}$ For this applied field value the vortex core is located just below the hole and the gyrotropic mode is resonantly excited, similar to the vortices in the other two disks. An increase of the static field leads to a shift of the vortex core towards the disk border and for higher fields to an increased asymmetric shape.

At $12.1 \mathrm{mT}$ the vortex describes an elliptical core trajectory with an asymmetric distortion of the vortex at the border of the sample, a "horseshoe" pattern is present in the differential images. Dependent on the delay time of the 187.5 MHz microwave excitation one obtains a black white black / black / white black white / white pattern instead of the crosses and coils if the vortex is located in the center or close to the center of the disk.

At present we cannot exclude the possibility that, besides the vortex gyrotropic mode, other contributions to the contrast changes obtained in this horseshoelike mode are present. Videos of the suppressed vortex gyrotropic mode $\left(B_{\text {ext }}=0 \mathrm{mT}\right)$ and the nucleated vortex core at $B_{\text {ext }}=4.3 \mathrm{mT}$ and at $B_{\text {ext }}=12.1 \mathrm{mT}$ are available as auxiliary material. ${ }^{24}$

Concerning the nature and the frequency of the vortex core gyrotropic motion in a static external field, a more comprehensive analysis of the vortex core dynamics requires a more detailed experimental and theoretical investigation. One could for instance study the complete saturation into a monodomain state by applying higher external static fields. This would help to investigate the evolution of the horseshoelike mode in more detail, as well as the annihilation and nucleation processes of the vortex and its subsequent resonant behavior in the subgigahertz regime. However, we want to stress that Novosad et al. have found only a weak field dependence in Permalloy disks. ${ }^{23}$ This changes in ellipses where a strong field dependence of the vortex gyrotropic mode frequency along the short axis of the ellipse has been reported recently. ${ }^{25}$ Furthermore it has been found that even in circular disks the vortex gyrotropic mode frequency is significantly influenced by intrinsic defects originating, e.g., from a crystallographic texture. ${ }^{26}$ The SR-FMR technique we used in the present work offers unique capabilities to study and image the intricate interplay between vortex core annihilation, nucleation, and the corresponding vortex gyrotropic mode behavior of magnetic vortices in the presence of one or more artificial small holes in ferromagnetic nanostructures directly.

In summary, we have investigated the vortex core motion in Permalloy disks in the presence of artificial defects in the form of small holes and studied their intercorrelation. We have demonstrated that the vortex core can be effectively captured within a hole which subsequently completely suppresses the vortex core motion in an exciting microwave field. With an applied static field of $4.3 \mathrm{mT}$ it was possible to nucleate the vortex core from the defect and excite a gyrotropic motion again. With increasing the applied field the vortex core gyration is displaced further from the center of the disk which in addition leads to an asymmetric vortex core motion.

Most of the work has been performed at the Advanced Light Source which is supported by the U.S. Department of Energy under Contract No. DE-AC03-76SF00098.

${ }^{1}$ C. Kittel, Rev. Mod. Phys. 21, 541 (1949).

${ }^{2}$ A. Hubert and R. Schäfer, Magnetic Domains (Springer, Berlin, 1998).

${ }^{3}$ T. Shinjo, T. Okuno, R. Hassdorf, K. Shigeto, and T. Ono, Science 289, 930 (2000).

${ }^{4}$ A. Wachowiak, J. Wiebe, M. Bode, O. Pietzsch, M. Morgenstern, and R. Wiesendanger, Science 298, 577 (2002).

${ }^{5}$ W. K. Hiebert, A. Stankiewicz, and M. R. Freeman, Phys. Rev. Lett. 79, 1134 (1997)

${ }^{6}$ Y. Acremann, C. H. Back, M. Buess, O. Portmann, A. Vaterlaus, D. Pescia, and H. Melchior, Science 290, 492 (2000).

${ }^{7}$ T. Gerrits, H. A. M. van den Berg, J. Hohlfeld, L. Bär, and T. Rasing, Nature (London) 418, 509 (2002).

${ }^{8}$ J. P. Park, P. Eames, D. M. Engebretson, J. Berezovsky, and P. A. Crowell, Phys. Rev. B 67, 020403 (2003).

${ }^{9}$ S.-B. Choe, Y. Acremann, A. Scholl, A. Bauer, A. Doran, J. Stöhr, and H. A. Padmore, Science 304, 420 (2004).

${ }^{10}$ H. Stoll, A. Puzic, B. Van Waeyenberge, P. Fischer, J. Raabe, M. Buess, T. Haug, R. Höllinger, C. Back, D. Weiss, and G. Denbeaux, Appl. Phys. Lett. 84, 3328 (2004).

${ }^{11}$ A. Puzic, B. Van Waeyenberge, K. W. Chou, P. Fischer, H. Stoll, G. Schütz, T. Tyliszczak, K. Rott, H. Brückl, G. Reiss, I. Neudecker, T. Haug, M. Buess, and C. H. Back, J. Appl. Phys. 97, 10E704 (2005).

${ }^{12}$ J. Raabe, C. Quitmann, C. H. Back, F. Nolting, S. Johnson, and C. Buehler, Phys. Rev. Lett. 94, 217204 (2005).

${ }^{13}$ J. P. Park and P. A. Crowell, Phys. Rev. Lett. 95, 167201 (2005).

${ }^{14}$ B. Van Waeyenberge, A. Puzic, H. Stoll, K. W. Chou, T. Tyliszczak, R. Hertel, M. Fähnle, H. Brückl, K. Rott, G. Reiss, I. Neudecker, D. Weiss, and C. H. Back, G. Schütz, Nature (London) 444, 461 (2006).

${ }^{15}$ A. R. Pereira, L. A. Mól, S. A. Leonel, P. Z. Coura, and B. V. Costa, Phys. Rev. B 68, 132409 (2003).

${ }^{16}$ M. Rahm, J. Stahl, W. Wegscheider, and D. Weiss, Appl. Phys. Lett. 85, 1553 (2004).

${ }^{17}$ M. Rahm, R. Höllinger, V. Umansky, and D. Weiss, J. Appl. Phys. 95, 6708 (2004)

${ }^{18}$ T. Uhlig, M. Rahm, C. Dietrich, R. Höllinger, M. Heumann, D. Weiss, and J. Zweck, Phys. Rev. Lett. 95, 237205 (2005).

${ }^{19}$ L. Bischoff, Ultramicroscopy 103, 59 (2005).

${ }^{20}$ A. Kilcoyne, T. Tyliszczak, W. F. Steele, S. Fakra, P. Hitchcock, K. Franck, E. Anderson, B. Harteneck, E. Rightor, G. Mitchell, A. P. Hitchcock, L. Yang, T. Warwick, and H. Ade, J. Synchrotron Radiat. 10, 125 (2003)

${ }^{21}$ G. Schütz, W. Wagner, W. Wilhelm, P. Kienle, R. Zeller, R. Frahm, and G. Materlik, Phys. Rev. Lett. 58, 737 (1987).

${ }^{22}$ K. Y. Guslienko, X. F. Han, D. J. Keavney, R. Divan, and S. D. Bader, Phys. Rev. Lett. 96, 067205 (2006)

${ }^{23}$ V. Novosad, F. Y. Fradin, P. E. Roy, K. S. Buchanan, K. Y. Guslienko, and S. D. Bader, Phys. Rev. B 72, 024455 (2005).

${ }^{24}$ See EPAPS Document No. E-APPLAB-90-084705 for videos of the suppressed gyroptropic mode $\left(B_{\text {est }}=0 \mathrm{mT}\right)$, and of the gyrotropic mode of the nucleated vortex core at $B_{\text {est }}=4.3 \mathrm{mT}$, and $B_{\text {est }}=12.1 \mathrm{mT}$. This document can be reached via a direct link in the online article's HTML reference section or via the EPAPS homepage (http://www.aip.org/pubservs/ epaps.html).

${ }^{25}$ K. S. Buchanan, P. E. Roy, M. Grimsditch, F. Y. Fradin, K. Y. Guslienko, S. D. Bader, and V. Novosad, Phys. Rev. B 74, 064404 (2006).

${ }^{26}$ R. L. Compton and P. A. Crowell, Phys. Rev. Lett. 97, 137202 (2006). 